\title{
CARACTERIZAÇÃO DE EXTREMOS MENSAIS DE PRECIPITAÇÃO EM CACOAL (RO)
}

\author{
SANTOS, Roziane Sobreira dos- roziane.dsantos@gmail.com \\ Universidade Federal de Rondônia / UNIR
}

VIEIRA, Pablo Dias - pabforever.ok@gmail.com

Universidade Federal de Rondônia / UNIR

EVANGELISTA, Dilson Henrique Ramos - dilsonh@gmail.comUniversidade
Federal de Rondônia / UNIR

OLIVEIRA, Leydimere Janny Cota - leydimere@gmail.com

Universidade Federal do Pampa / UNIPAMPA

NONATO, Deivisom - deivisom.nonato@gmail.com

Universidade Federal de Rondônia / UNIR

\begin{abstract}
RESUMO: Este estudo teve por objetivo ajustar uma distribuição de probabilidade a dados de precipitação extrema mensal em uma região no sudoeste da Amazônia, visando uma melhor compreensão da dinâmica dos extremos de precipitação, por meio de modelagem estatística, fornecendo subsídios para análises e planejamento futuro. A estimação dos parâmetros de cada distribuição candidata foi realizada utilizando o método de máxima verossimilhança, que apresenta bons resultados com variância menor das estimativas. Para a aderência dos dados às distribuições propostas foi realizada a verificação por métodos gráficos (diagramas de QQ-plot e PP-Plot) e utilizado o teste de aderência de Kolmogorov-Smirnov com $5 \%$ de significância. Os resultados mostraram que a distribuição extrema de chuvas em Cacoal é em torno de $50 \mathrm{~mm}$. A análise gráfica apontou para bom ajuste das distribuições estudadas, com exceção da distribuição Exponencial. Ao realizar o teste de aderência de Kolmogorov-Smirnov com $5 \%$ de significância, constatou-se o observado graficamente, sendo que as distribuições Lognormal, seguida da distribuição Gumbel, se apresentaram como as distribuições que melhor se ajustaram aos dados em análise.
\end{abstract}

PALAVRAS-CHAVES: Precipitação extrema, Ajuste probabilístico, Estimativas de parâmetros.

\section{CHARACTERIZATION OF MONTHLY PRECIPITATION EXTREMES IN CACOAL (RO)}

ABSTRACT: This study aimed to adjust a probability distribution to extreme monthly precipitation data in a region in the southwest of the Amazon, aiming at a better understanding of the dynamics of precipitation extremes, through statistical modeling, providing subsidies for analysis and future planning. The estimation of the parameters of each candidate distribution will be performed using the maximum likelihood method, which presents good results with a smaller variance of the estimates. For the adherence of the data to the proposed distributions, the verification was performed by graphical methods (QQ-plot and PP-Plot diagrams) and the Kolmogorov-Smirnov adhesion test was used with $5 \%$ significance. The results showed that the extreme distribution of rainfall in Cacoal is around $50 \mathrm{~mm}$. The graphical analysis pointed to good adjustments of the studied distributions, with the exception of the Exponential distribution. When performing the adherence test with $5 \%$ of Kolmogorov-Smirnov significance, we found the graphically observed, and the Lognormal distributions, followed by the Gumbel distribution, presented as the distributions that best fit the data under analysis.

KEYWORDS: Extreme Precipitation, Probabilistic Adjustment, Parameter Estimates 
CARACTERIZACIÓN DE EXTREMOS MENSAJES DE PRECIPITACIÓN EN CACOAL $(R O)$

RESUMEN: Este estudio tuvo por objetivo ajustar una distribución de probabilidad a datos de precipitación extrema mensual en una región en el suroeste de la Amazonia, buscando una mejor comprensión de la dinámica de los extremos de precipitación, por medio de modelado estadístico, proporcionando subsidios para análisis y planificación futura. La estimación de los parámetros de cada distribución candidata fue realizada utilizando el método de máxima verosimilitud, que presenta buenos resultados con varianza menor de las estimativas. Para la adherencia de los datos a las distribuciones propuestas se realizó la verificación por métodos gráficos (diagramas de QQ-plot y PP-Plot) y se utilizó la prueba de adherencia de Kolmogorov-Smirnov con un $5 \%$ de significancia. Los resultados mostraron que la distribución extrema de Iluvias en Cacoal es alrededor de $50 \mathrm{~mm}$. El análisis gráfico apuntó para un buen ajuste de las distribuciones estudiadas, con excepción de la distribución Exponencial. Al realizar la prueba de adherencia de Kolmogorov-Smirnov con un $5 \%$ de significancia, se constató el observado gráficamente, siendo que las distribuciones Lognormal, seguida de la distribución Gumbel, se presentaron como las distribuciones que mejor se ajustaron a los datos en análisis.

PALABRAS CLAVE: Precipitación extrema, Ajuste probabilístico, Estimaciones de parámetros.

\section{CARACTÉRISATION DE LA PRÉCIPITATION MENSUELLE EXTREMES DANS CACOAL (RO)}

RÉSUMÉ: Cette étude visait à ajuster une distribution de probabilité aux données des précipitations mensuelles extrêmes dans une région du sud-ouest de l'Amazonie, en vue de mieux comprendre la dynamique des précipitations extrêmes, par la modélisation statistique, en fournissant des subventions pour l'analyse et la planification future. L'estimation des paramètres de chaque distribution candidate a été effectuée en utilisant la méthode du maximum de vraisemblance, qui présente de bons résultats avec une variance plus faible des estimations. Pour l'adhésion des données aux distributions proposées, la vérification a été effectuée par des méthodes graphiques (diagrammes QQ-plot et PP-Plot) et le test d'adhésion de Kolmogorov-Smirnov a été utilisé avec une signification de $5 \%$. Les résultats ont montré que la distribution extrême des précipitations à Cacoal est d'environ $50 \mathrm{~mm}$. L'analyse graphique a montré un bon ajustement des distributions étudiées, à l'exception de la distribution exponentielle. Lorsque le test d'adhésion de Kolmogorov-Smirnov a été réalisé avec une signification de $5 \%$, il a été observé que les distributions lognormales, suivies de la distribution de Gumbel, étaient présentées comme les distributions les mieux adaptées aux données analysées.

MOTS CLÉS: Précipitation extrême, Ajustement probabiliste, Estimations paramétriques

\section{INTRODUÇÃO}

A informação climática é de grande importância nas diversas atividades realizadas pelo homem, como o gerenciamento de recursos hídricos, proteção 
aos riscos de inundação, modelagem da erosão, estudos agrometeorológicos, otimização da produção agropecuária, estudos climáticos de fenômenos atmosféricos, mudanças climáticas, dentre outras aplicações.

Dentre os elementos climáticos, a precipitação é um dos que exerce influência expressiva, pois, tanto o excesso como a escassez causam grandes transtornos ambientais e socioeconômicos. É fundamental para modelagem hidrológica, modelos agrícolas e estudos de impactos climáticos (KLEIBER et al., 2012).

Compreender eventos extremos de precipitação em uma região é muito importante, pois os mesmos têm grande potencial para causar danos materiais e perdas de vidas humanas (BLAIN e CAMARGO, 2012). Eventos de precipitação extrema causam um grande impacto sobre as atividades socioeconômicas da Amazônia, tornando a população vulnerável ao comportamento e à variabilidade do sistema climático (SANTOS et al., 2016).

Em 2014, Acre e Rondônia declararam um estado de calamidade devido a inundações causadas por fortes chuvas nas nascentes dos rios (SANTOS et al., 2016). Cotta et al. (2016) destacam a importância de estudar extremos de precipitação objetivando informações como diagnóstico de impacto, frequência e previsões para minimizar os impactos na população local.

Analisar a probabilidade da ocorrência de eventos de precipitação é importante para o planejamento de atividades expostas aos seus efeitos adversos (SANTOS et al., 2016), como planejamento de atividades relacionadas à agricultura ou no monitoramento de processos hidrológicos concernentes às bacias hidrográficas (KIST e VIRGENS FILHO, 2015).

A precipitação é considerada um processo hidrológico estocástico, ou regida por leis de probabilidades, já que é influenciada pelas estações do ano ou pela radiação solar no topo da atmosfera ao longo da órbita da Terra em torno do Sol, que são componentes aleatórios (NAGHETTINI e PINTO, 2007), desta forma pode se aplicar as leis probabilísticas, e ajustar um modelo de distribuição de probabilidade.

Ajustar uma distribuição de probabilidade aos dados de precipitação possibilita compreender os padrões de ocorrência e permite conhecer qual a probabilidade de ocorrência de determinada quantidade de chuva em uma localidade, de forma antecipada, permitindo o planejamento estratégico e programação de ações que venham minimizar os impactos que podem vir a ser causados, auxiliando a tomada de decisão de gestores e governantes.

O ajuste de uma distribuição de probabilidade consiste em encontrar uma função que represente a variável aleatória (BESKOW et al., 2015). A escolha e a definição da distribuição que melhor se ajusta ao conjunto de dados em análise devem ser realizadas comparando-se as frequências teóricas às frequências observadas por meio de testes estatísticos não paramétricos (MELLO e SILVA, 2013).

No Brasil, muitos pesquisadores têm se empenhado nos estudos de ajuste de distribuições probabilísticas a dados de precipitação extrema. Neto et al. (2005) comparando o ajuste das funções de densidade de probabilidade Gama, Exponencial, Weibull, Normal e Log-Normal em dados diários de 
precipitação pluvial em Piracicaba-SP, concluíram que as distribuições Gama seguida pela Weilbul, obtiveram melhores performance.

Resultados semelhantes foram encontrados por Kist e Virgens Filho (2015), na análise de dados diários de chuva em localidades do Paraná quando não considerou a distribuição Exponencial Mista.

Souza et al. (2015) ajustaram as distribuições Normal, Log-Normal, Exponencial, Gama, Gumbel, Weibull e Logística para os eventos de precipitação extrema em diversas localidades de Rondônia, para estimação da chamada função Intensidade-Duração-Frequência (IDF), e notou-se que a distribuição Gumbel tem maior aplicabilidade ao fenômeno de chuvas extremas.

Estudos para a caracterização probabilística de chuvas extremas no Estado Rondônia, localizado no sudoeste da Amazônia, são extremamente poucos, pois é praticamente desprovido de informações de chuvas intensas (SOUZA et al., 2015).

Diante do exposto, objetivou-se com este estudo ajustar uma distribuição de probabilidade a dados de precipitação extrema mensal em uma região no sudoeste da Amazônia, visando uma melhor compreensão da dinâmica dos extremos de precipitação, por meio de modelagem estatística, fornecendo subsídios para análises e planejamento futuro.

Material e Métodos

Nesse estudo, foram utilizados dados mensais de precipitação da estação meteorológica de superfície automática de Cacoal, localizada no sudoeste da Amazônia brasileira, na latitude $-11,45^{\circ}$ sul e longitude $-61,43^{\circ}$ oeste, a 184 metros de altitude, pertencente à rede de estações do Instituto Nacional de Meteorologia (INMET), no estado de Rondônia.

De acordo com a classificação Koppen o clima da região é tipo Aw, com duas estações bem definidas verão chuvoso e inverno seco, mais informações podem ser obtidas em Barbosa (2012).

Foram considerados os valores máximos mensais de precipitação, compreendidos no período de 2008 a 2016, nos principais meses chuvosos da região: outubro, novembro, dezembro, janeiro, fevereiro, março e abril (FURLAN, 2009). Ao todo foram utilizadas 53 observações de máximos mensais de precipitação. Em Rondônia a precipitação é de monções, ocorrendo somente quando o continente é aquecido acima dos níveis de temperatura da superfície do oceano (BUTT et al., 2011).

Inicialmente foi realizada uma análise exploratória dos dados, empregando estatísticas descritivas e técnicas gráficas (MORETTIN e BUSSAB, 2010), para caracterizar o comportamento, a forma e tipo de função densidade de probabilidade que poderiam ser utilizadas para ajustar aos dados de precipitação máxima mensal.

Para identificar a função densidade de probabilidade que melhor se ajusta aos dados de precipitação máxima mensal, deve-se considerar a distribuição e propriedades estatísticas dos mesmos. Os dados de precipitação comumente apresentam assimetria, geralmente são modelados por famílias de distribuições que são assimétricas à direita, ou positiva (HUNDECHA et al., 2009). 
O ajuste de distribuições de probabilidade em dados hidrológicos tem sido objeto de estudo em várias localidades no Brasil, entre alguns autores que estudaram o tema destacam-se: Borges e Thebaldi (2016); Beskow et al. (2015); Souza et al. (2015); Franco et al. (2014); Catalunha et al. (2002). Sendo as distribuições Exponencial, Gama, Gumbel, Lognormal e Weibull, muito utilizadas. Dessa forma, considerando os dados em análise e a literatura consultada, neste estudo foram analisadas as distribuições supracitadas.

Tabela 1 - Distribuições de probabilidades e suas respectivas funções densidade de probabilidade.

\begin{tabular}{cl}
\hline $\begin{array}{c}\text { Distribuição de } \\
\text { Probabilidade }\end{array}$ & Função Densidade de Probabilidade \\
\hline Exponencial $(\lambda)$ & $\lambda \mathrm{e}^{-\lambda x}, \mathrm{x} \geq 0 ; \lambda>0$ \\
Gama $(a, \beta)$ & $\frac{1}{\Gamma(a) \beta^{\alpha}} X^{\alpha-1} \mathrm{e}^{-\frac{x}{\beta}}, x>0 ; a>0, \beta>0$ \\
Weibull $(a, \beta)$ & $\frac{\alpha}{\beta^{\alpha}} x^{\alpha-1} \mathrm{e}^{-\left(\frac{x}{\beta}\right)^{\alpha}}, x>0 ; a>0, \beta>0$ \\
Log-Normal $(\mu, \sigma)$ & $\frac{1}{x \sigma \sqrt{2 \pi}} \mathrm{e}^{\frac{(\ln (x)-\mu)^{2}}{2 \sigma^{2}}} ; x>0 ;-\infty<\mu<\infty, \sigma>0$ \\
Gumbel $(a, \beta)$ & $\frac{1}{\alpha} \mathrm{e}^{-\left(\frac{x-\alpha}{\beta}\right)-\mathrm{e}^{-\left(\frac{x-\alpha}{\beta}\right)},-\infty<x<\infty ; a \in \mathbb{R}, \beta>0}$ \\
\hline
\end{tabular}

A estimação dos parâmetros de cada distribuição candidata foi realizada utilizando o método de máxima verossimilhança, que apresenta bons resultados com variância menor das estimativas (FISCHER et al., 2012). As estimativas foram calculadas com os dados observados (FRANCO et al., 2014).

A aderência dos dados às distribuições propostas foi verificada por métodos gráficos, com diagramas de QQ-plot (quantil-quantil) e PP-Plot (probabilidade-probabilidade). Estes diagramas comparam as distribuições empíricas e teóricas ajustadas em termos dos quantis empíricos (MONTGOMERY e RUNGER, 2012).

Após as análises descritivas foi empregado teste estatístico para indicar se os dados provêm ou não da distribuição em análise. O teste estabelece as seguintes hipóteses:

$\mathrm{H}_{0}$ : Os dados são consistentes com a distribuição especificada

$\mathrm{H}_{1}$ : Os dados não são consistentes com a distribuição especificada

O teste não-paramétrico de Kolmogorov-Sminorv (KS) é amplamente utilizado na literatura para verificar o ajuste de distribuições empíricas em hidrologia (CALDEIRA et al., 2015; FRANCO et al., 2014; ARAGÃO et al., 2013; FISCHER et al., 2012; BACK et al., 2011). 
O teste KS avalia a concordância entre a distribuição observada da amostra e uma determinada distribuição teórica. Verificando se os dados amostrais se aproximam razoavelmente de uma determinada distribuição.

O teste KS é baseado na distância entre a função de distribuição acumulada empírica $\left(F_{n}(x)\right)$ dos dados e a função de distribuição acumulada teórica $(F(x))$ de cada distribuição candidata (CORDER e FOREMAN, 2009; SU et al., 2009; HOLLANDER et al., 2014). A estatística do teste é o limite superior das diferenças em valor absoluto entre $F(x)$ e $F_{n}(x)$, conforme a equação (1) a seguir:

$$
\mathrm{D}_{\mathrm{n}}=\max _{\mathrm{x} \in \mathrm{R}}\left|\mathrm{F}_{\mathrm{n}}(\mathrm{x})-\mathrm{F}(\mathrm{x})\right|
$$

Wilks (2006) pontua que para distribuições contínuas, o teste de Kolmogorov-Smirnov é adequado para estas análises. Neste trabalho foi adotado um nível de significância de $5 \%$ para o teste.

O QQ-plot e o teste de Kolmogorov-Smirnov comparam as distribuições empíricas acumuladas com as teóricas esperadas, estas mesmas técnicas foram utilizadas por Santos et al. (2016) para avaliar o ajuste da distribuição de valor extremo generalizada e da distribuição generalizada de Pareto a extremos diários de precipitação na Amazônia Brasileira.

Os dados foram organizados e analisados em conjunto de planilha eletrônica com o software livre $R$ ( $R$ CORE TEAM, 2017). O ajuste das distribuições de probabilidades foi feito pelo pacote fitdistrplus (DELIGNETTEMULLER e DUTANG, 2015).

\section{RESULTADOS E DISCUSSÃO}

Os valores de precipitação máxima mensal para os meses de outubro a abril, durante 2008 a 2016, totalizando 53 observações, para a cidade de Cacoal - RO são apresentados no histograma juntamente com a curva de densidade empírica (Figura 1). Os dados são divididos em classe, qual se contabiliza as respectivas frequências relativas e plotadas no histograma conforme descrito por Morettin e Bussab (2010). Observa-se uma assimetria a direita, com valor mediano um pouco menor do que a média, com agrupamento dos dados mais na esquerda da distribuição. Em geral, este comportamento é observado em dados de precipitação (COTTA et al., 2016; MELLO e OLIVEIRA, 2016; LUNDGREN et al., 2015; THEBALDI, 2012; RONDON, 2001). 


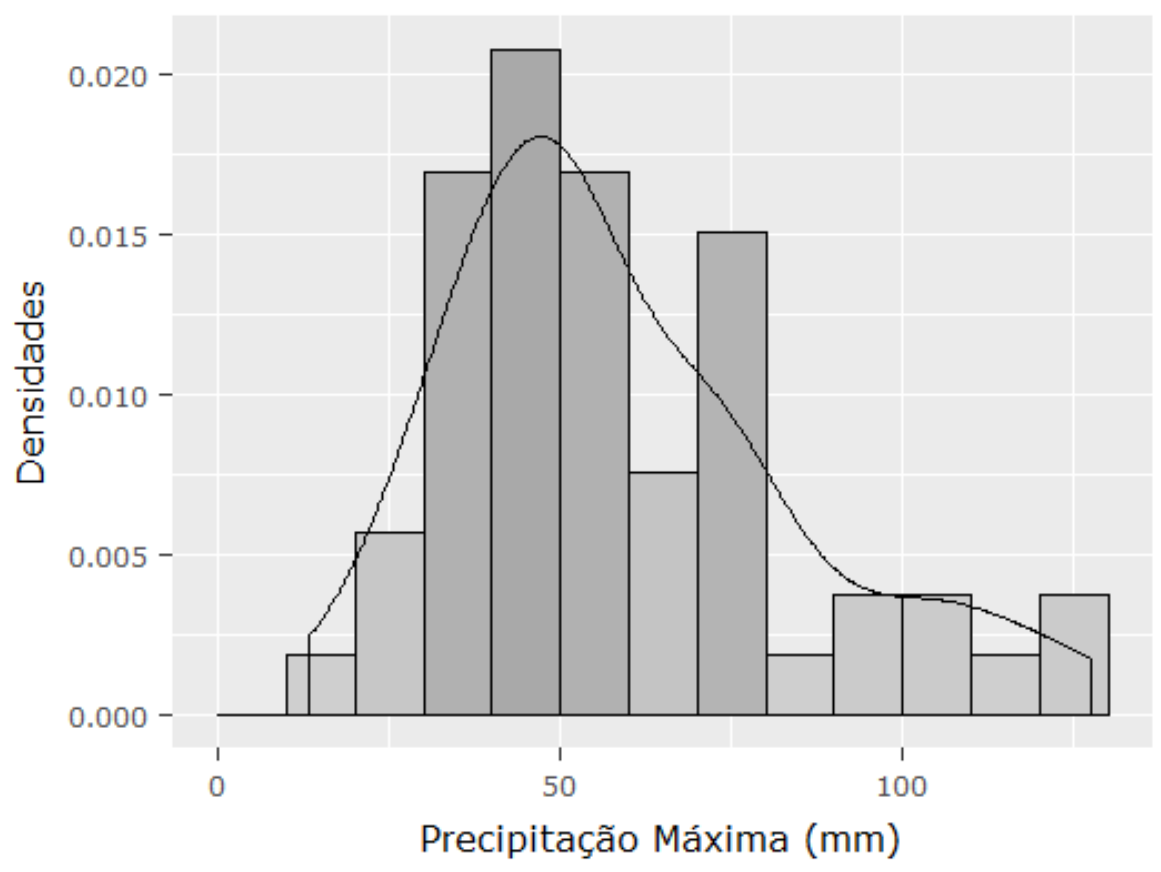

Figura 1 - Frequências de ocorrência de precipitação extrema em Cacoal, RO.

Ressalta-se uma concentração em torno do valor de $50 \mathrm{~mm}$. A Tabela 2 apresenta as estatísticas descritivas para a série de dados estudada, totalizando $\mathrm{n}=53$ observações. O valor máximo de precipitação observado, no período de 2008 a 2016 em estudo, foi de 127,6 milímetros, ocorrido em março de 2014 e o mínimo de 13,4 mm, observado em abril de 2016.

Tabela 2 - Estatísticas descritivas da precipitação máxima nos meses de outubro a abril

\begin{tabular}{ccccccc}
\hline $\begin{array}{c}\text { Mínimo } \\
(\mathbf{m m})\end{array}$ & $\begin{array}{c}\text { Máximo } \\
\mathbf{( \mathbf { m m } )}\end{array}$ & $\begin{array}{c}\text { Média } \\
\mathbf{( \mathbf { m m } )}\end{array}$ & $\begin{array}{c}\text { Mediana } \\
\mathbf{( \mathbf { m m } )}\end{array}$ & $\begin{array}{c}\text { Desvio } \\
\text { Padrão } \\
\mathbf{( \mathbf { m m } )}\end{array}$ & Assimetria & Curtose \\
\hline 13,4 & 127,6 & 58,9 & 52,2 & 25,6 & 0,872 & 3,397 \\
\hline
\end{tabular}

Os valores de assimetria positiva e curtose não são muito distantes de 3 (Tabela 2) (valor padrão para a distribuição normal), indicando o ajuste de distribuições comuns com assimetria positiva, como as distribuições Weibull, Gama, Gumbel e Lognormal (Figura 2).

A Figura 2 apresenta o gráfico descritivo de assimetria e curtose proposto por Cullen e Frey (1999) de ajuste de distribuição, que fornece uma indicação de qual distribuição pode se ajustar aos dados. Nesta plotagem, os valores das distribuições comuns são exibidos para ajudar na escolha das distribuições para os dados. Para algumas distribuições (Normal, Uniforme, Logística, Exponencial), existe apenas um valor possível para a assimetria e a curtose. Assim, a distribuição é representada por um único ponto no gráfico. Para outras distribuições, áreas de valores possíveis são representadas, consistindo em 
linhas (como para as distribuições Gama e Lognormal), ou áreas maiores (como para a distribuição Beta) (DELIGNETTE-MULLER e DUTANG, 2015).

Avaliando os possíveis ajustes das distribuições, descritivamente (Figura 2), observa-se uma assimetria positiva e curtose não muito distante de 3 , indicando que o ajuste de distribuições com assimetria a direita poderá ser considerado, como a Gama, Lognormal e Weibull são mais próximas dos dados, ilustrando que a distribuição gama pode se ajustar bem a série de precipitação máxima.

Destaca-se ainda que a distribuição exponencial está muito distante, indicando não ser uma boa distribuição candidata ao ajuste da precipitação máxima (Figura 2). Nesse gráfico não foi apresentada a distribuição Gumbel.

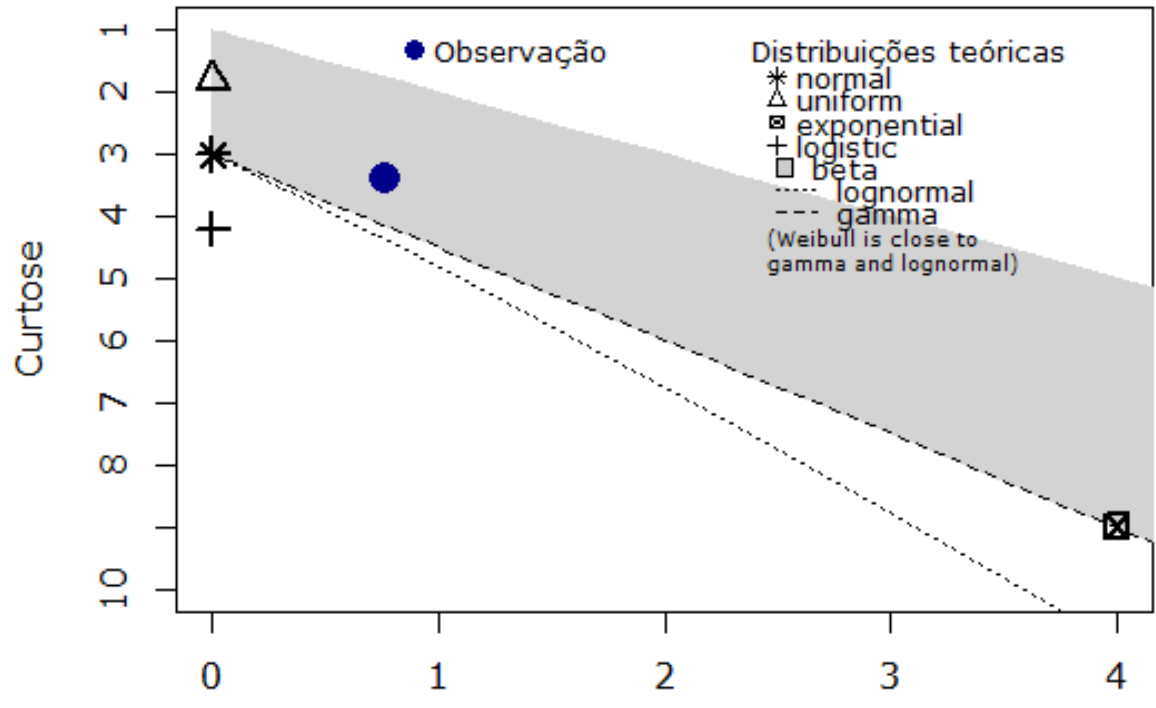

Quadrado de assimetria

Figura 2 - Gráfico de assimetria e curtose com as distribuições a serem ajustadas.

Na Figura 3 são apresentados os gráficos de histograma juntamente com os ajustes das funções de probabilidade, as densidades empíricas acumuladas e os gráficos QQ-plot e PP-plot. Pela análise do histograma, observa-se que as distribuições Gumbel, Lognormal e Gama são as que melhor se ajustam às variações nos extremos de precipitação.

O modelo Gumbel foi considerado bem ajustado a dados de precipitação máxima em algumas localidades de Minas Gerais, cita-se: Borges e Thebaldi (2016) estudando o ajuste de modelos estocásticos na predição da precipitação máxima diária anual no município de Formiga MG, os quais constataram que o modelo Gumbel apresentou maior adesão; Thebaldi (2012), também observou o ajuste do referido modelo ao analisar precipitação máxima diária anual do município de Uberaba, MG. Já Caldeira et al. (2015), ajustaram diferentes modelos probabilísticos às séries de precipitação máxima diária anual no Rio Grande do Sul, entre eles o Log-Normal com 2 parâmetros, Log-Normal com 3 
parâmetros (LN-3P) e Gumbel, resultando os melhores ajustes pela distribuição Log-Normal de 3 parâmetros.

Os gráficos QQ-plot e PP-plot são utilizados para analisar a falta de ajustes nas caudas e no centro das distribuições, respectivamente. Todas as distribuições ajustadas apresentam falta de ajuste no centro (PP-plot), sendo a distribuição Lognormal, juntamente com a Gumbel e Gama as que apresentam os melhores ajustes para o centro (Figura 3). Com relação ao ajuste nas caudas (QQ-Plot), as distribuições Gama, Gumbel e Weibull foram as que apresentaram os melhores ajustes (Figura 3).

Em todos os gráficos, observa-se que a distribuição exponencial não se ajusta bem aos dados, indicando não ser adequada para dados de precipitação extrema (Figura 3).

Dados de precipitação máxima apresentam características assintóticas, ou seja, com acúmulo de dados à esquerda na distribuição de frequências, dessa forma a análise do ajuste nas caudas da distribuição é muito importante.
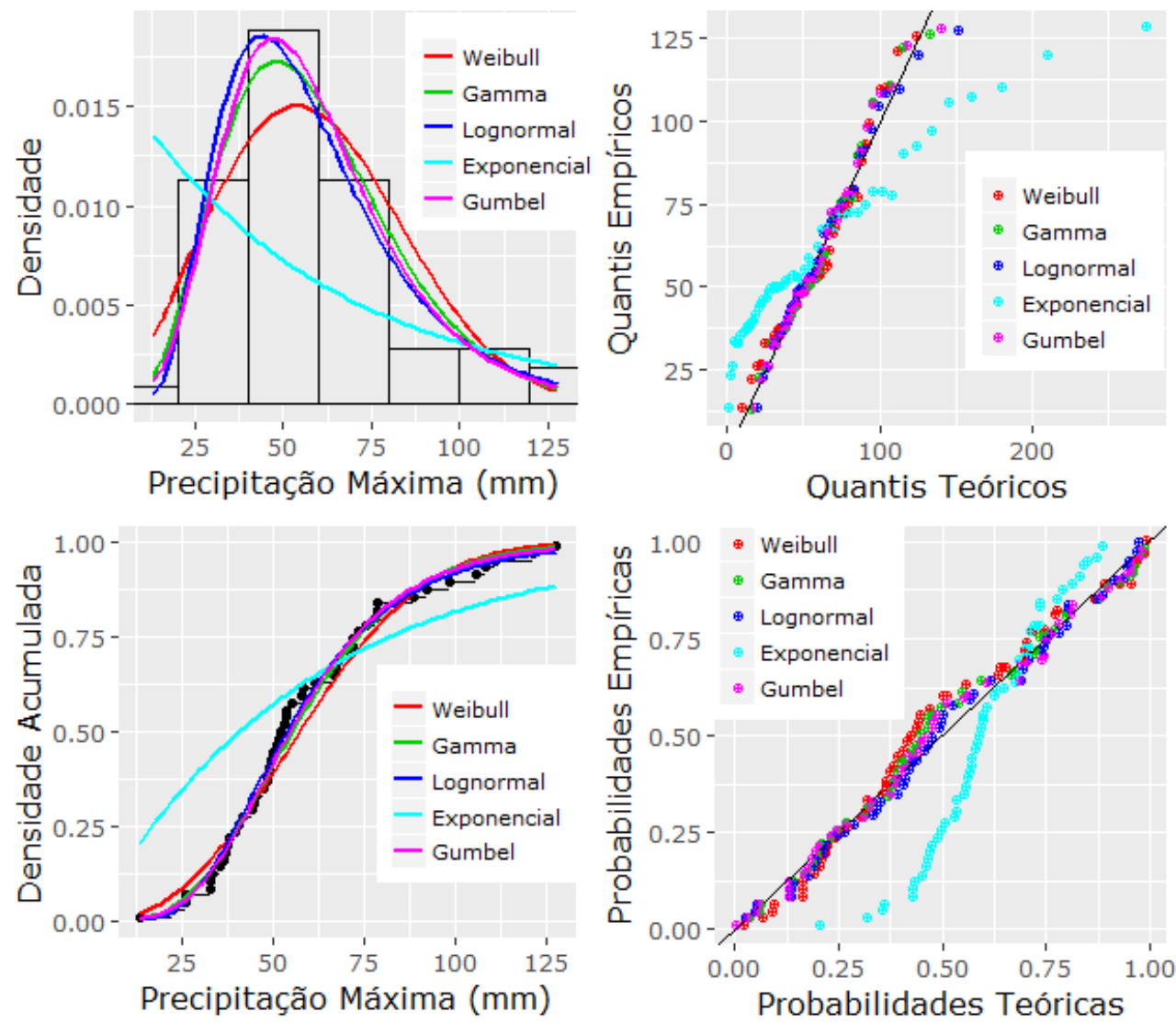

Figura 3 - Ajustes das distribuições teóricas para precipitação máxima de Cacoal, RO: a) Histograma e densidades de probabilidades, b) Q-Q plot, c) Densidades acumuladas, d) P-P plot.

A Tabela 3 apresenta as estimativas dos parâmetros, juntamente com o respectivo erro padrão, para cada distribuição analisada. 
Tabela 3 - Estimativas dos parâmetros das distribuições

\begin{tabular}{llccc}
\hline Distribuição & $\begin{array}{c}\text { Parâmetro } \\
\text { Estimado }\end{array}$ & $\begin{array}{c}\text { Erro } \\
\text { Padrão }\end{array}$ & $\begin{array}{c}\text { Parâmetro } \\
\text { Estimado }\end{array}$ & $\begin{array}{c}\text { Erro } \\
\text { Padrão }\end{array}$ \\
\hline Weibull & $a=66,53$ & 3,93 & $\beta=2,46$ & 0,25 \\
Gama & $a=0,09$ & 0,02 & $\beta=5,51$ & 1,04 \\
Lognormal & $\mu=3,98$ & 0,06 & $\sigma=0,44$ & 0,04 \\
Gumbel & $a=19,98$ & 2,15 & $\beta=47,3$ & 2,89 \\
Exponencial & $\lambda=0,02$ & 0,002 & - & - \\
\hline
\end{tabular}

O teste de aderência de Kolmogorov-Smirnov é utilizado para verificar estatisticamente, em conjuntos com as análises gráficas anteriores, a distribuição de probabilidade que melhor se adéqua aos dados de precipitação máxima na região de Cacoal (Tabela 4).

Analisando o p-valor das distribuições, ao nível se $5 \%$, verifica-se que apenas a distribuição exponencial não foi considerada apta para o ajuste da série de precipitação máxima mensal (Tabela 4). Dessa forma, há indícios estatísticos de que as demais distribuições de ajustam aos dados.

Tabela 4 - Teste de aderência de Kolmogorov-Smirnov

\begin{tabular}{ccc}
\hline Distribuição & D & p-valor \\
\hline Gama & 0,0838 & 0,8288 \\
Weibull & 0,1191 & 0,4397 \\
LogNormal & 0,0633 & 0,9838 \\
Gumbel & 0,0803 & 0,8833 \\
Exponencial & 0,3535 & 0,0000 \\
\hline
\end{tabular}

Considerando a verificação e os ajustes dos modelos probabilísticos à série de precipitação máxima e os resultados do teste de Kolmogorov-Smirnov, constatou-se, ao nível de 5\%, que a distribuição exponencial não é adequada. Catalunha et al. (2002), ao testar o ajuste de distribuição em dados de precipitação em Minas Gerais, também observou a falta de ajuste da mesma distribuição.

Naghettini e Pinto (2007) apontam que o teste de aderência é apenas uma das técnicas que deve ser utilizadas para analisar o ajuste de uma distribuição de probabilidade a dados hidrológicos.

As análises descritivas e gráficas (Figura 3) e o teste de KolmogorovSmirnov, indicam as distribuições Lognormal, Gumbel, Gama e Weibull, nessa ordem, são plausíveis para uso na modelagem de eventos extremos de precipitação no sudoeste da Amazônia na região de Cacoal.

Os resultados encontrados neste estudo, estão em consonância com os encontrados por Souza et al. (2015) e Souza (2012), que estudaram os ajustes de distribuições teóricas de probabilidade no estado de Rondônia.

Com relação a estatística D do teste de KS (Tabela 4), menores valores da estatística $D$ fornecem maiores valores de $p$-valor e, isto é, maior aderência dos dados à distribuição de probabilidade em análise (CARGNELUTTI FILHO et 
al., 2004). Dessa forma, a distribuição Lognormal, seguida da distribuição Gumbel, se apresentam como as distribuições que melhor se ajustaram aos dados em análise.

Souza et al. (2015) destacaram ainda que a distribuição Lognormal aplicada às probabilidades dos dados pluviométricos analisados se encontraram dispostos nos núcleos das curvas de distribuições e não em suas caudas, indicando que a distribuição Gumbel seria mais adequada para a modelagem de eventos extremos de precipitação em Rondônia.

Cabe ressaltar que, para uma análise de gerenciamento de recursos hídricos, caracterização de enchentes e secas, dentre outras aplicações, é necessárias séries longas de precipitação. No entanto, os registros históricos de precipitação com resolução espacial e temporal desejada são quase sempre insuficientes (LI et al., 2012).

Li et al. (2012) afirmaram que é difícil quantificar a incerteza dos resultados do modelo com apenas uma série de dados. Entretanto, há uma preocupação de que, mudanças climáticas, podem estar aumentando as frequências e as magnitudes dos extremos de precipitação, justificando a necessidade de se conhecer e analisar esses extremos, mesmo com poucos dados.

\section{CONCLUSÕES}

A modelagem probabilística proposta neste trabalho para os valores extremos de precipitação entre os meses de outubro a março no Município de Cacoal mostrou que as distribuições Lognormal, Gumbel, Gama e Weibull obtiveram ajuste satisfatório no período estudado. Nesse sentido a distribuição de probabilidade Lognormal, seguida pela Gumbel mostraram-se mais adequadas para ajuste à série de dados de precipitação extrema de Cacoal e, de maneira oposta a distribuição Exponencial não se mostrou adequada para esta modelagem.

\section{REFERÊNCIAS BIBLIOGRÁFICAS}

ARAGÃO, R.; SANTANA, G. R.; COSTA, C. E. F. F.; CRUZ, C. E. F. F.; FIGUEIREDO, E. E.; SRINIVASAN, V. S. Chuvas intensas para o estado de Sergipe com base em dados desagregados de chuva diária. Revista Brasileira de Engenharia Agrícola e Ambiental, v. 17, n. 3, p. 243-252, 2013.

BACK, A. J.; HEN, A.; OLIVEIRA, J. L. R. Heavy rainfall equations for Santa Catarina, Brazil. Revista Brasileira de Ciência do Solo, v. 35, p. 2127-2134, 2011.

BARBOSA, L. S. Análise da qualidade da água e o processo de uso e ocupação das terras na bacia hidrográfica do rio Pirarara no município de Cacoal Rondônia. Dissertação (Mestrado em Geografia). Programa de Pós-GraduaçãoMestrado em Geografia da Fundação Universidade Federal de Rondônia (UNIR) 151p. Porto Velho, 2012.

BeSKOW, S.; CALDEIRA, T. L.; MELlO, C. R.; FARIA, L. C.; GUEDES, H. A. S. Multiparameter probability distributions for heavy rainfall modeling in extreme 
southern Brazil. Journal of Hydrology: Regional Studies, v. 4, Part B, p. 123133, 2015.

BLAIN, G. C.; CAMARGO, M. B. P. Probabilistic structure of an annual extreme rainfall series of a coastal area of the state of São Paulo, Brazil. Engenharia Agrícola, v. 32, n. 3, p. 552-559, 2012.

BORGES, G. M. R.; THEBALDI, M. S. Estimativa da precipitação máxima diária anual e equação de chuvas intensas para o município de Formiga, MG, Brasil. Revista Ambiente \& Água, v. 11, n. 4, 2016.

BUTT, N.; OLIVEIRA, P. A.; HEIL COSTA, M. H. Evidence that deforestation affects the onset of the rainy season in Rondônia, Brazil. Journal of Geophysical Research, v. 116, n. 11120, p. 1-8, 2011.

CALDEIRA, T. L.; BESKOW, S.; MELLO, C. R.; FARIA, L. C.; SOUZA, M. R.; GUEDES, H. A. S. Modelagem probabilística de eventos de precipitação extrema no estado do Rio Grande do Sul. Revista Brasileira de Engenharia Agrícola e Ambiental, v. 9, n. 3, p. 197-203, 2015.

CARGNELUTTI FILHO, A.; MATZENAUER, R.; TRINDADE, J. K. Ajustes de funções de distribuição de probabilidade à radiação solar global no Estado do Rio Grande do Sul. Pesquisa Agropecuária Brasileira, v.39, n. 12, p. 1157-1166, 2004.

CATALUNHA, J. M.; SEDIYAMA, G. C.; RIBEIRO, A.; LEAL, B. G.; SOARES, C. P. B. Aplicação de cinco funções densidade de probabilidade a série de precipitação pluvial do estado de Minas Gerais. Revista Brasileira de Agrometeorologia, Sete Lagoas, v. 10, n. 1, p. 153-162, 2002.

CORDER, G. W.; FOREMAN, D. I. Nonparametric Statistics for Non-Statisticians: A Step-by-Step Approach. John Wiley and Sons, 2009, 264 p.

COTTA, H. H. A.; CORREA, W. S. C.; AlBuQUeRQUE, T. T. A. Aplicação da distribuição de Gumbel para valores extremos de precipitação no município de Vitória-ES. Revista Brasileira de Climatologia, v. 19, 2016.

CULLEN, A. C.; FREY, H. C. Probabilistic Techniques in Exposure Assessment: A Handbook for Dealing with Variability and Uncertainty in Models and Inputs. 1 ed. Plenum Publishing Co Press: New York, 1999.

DELIGNETTE-MULLER, M. L.; DUTANG, C. Fitdistrplus: An R Package for Fitting Distributions. Journal of Statistical Software, 64 (4), 1-34, 2015. URL: <http://www.jstatsoft.org/v64/i04/>.

FISCHER, T.; LUO, B. S., Y.; SCHOLTEN, T. Probability Distribution of Precipitation Extremes for Weather Index-Based Insurance in the Zhujiang River Basin, South China. Journal of Hydrometeorology, v. 13, p. 1023-1037, 2012.

FRANCO, C. S.; MARQUES, R. F. P. V.; OliveIRA, A. S.; OliveirA, L. F. C. Distribuição de probabilidades para precipitação máxima diária na Bacia Hidrográfica do Rio Verde, Minas Gerais. Revista Brasileira de Engenharia Agrícola e Ambiental, v. 18, n. 7, p. 735-741. 2014.

FURLAN, D. N. Variabilidade temporal e espacial das chuvas e do balanço hídrico no estado de Rondônia: caracterização e análise de tendência. 129p, 2009. Dissertação (Mestrado em Agronomia) - Escola Superior de Agricultura Luiz de Queiroz, Universidade de São Paulo. São Paulo, 2009. 
HOLLANDER, M.; WOLFE, D. A.; CHICKEN, E. Nonparametric statistical methods. $3^{a}$ ed, John Wiley \& Sons, 2014, 844p.

HUNDECHA, Y.; PAHLOW, M.; SCHUMANN, A. Modeling of daily precipitation at multiple locations using a mixture of distributions to characterize the extremes, Water Resources Research, v. 45, W12412, DOI:10.1029/ 2008WR007453. 2009.

KIST, A.; VIRGENS FILHO, J. S. Análise probabilística da distribuição de dados diários de chuva no estado do Paraná. Revista Ambiente \& Água, vol. 10, n. 1, p. $172-181,2015$.

KLEIBER, W., R. W. KATZ, AND B. RAJAGOPALAN. Daily spatiotemporal precipitation simulation using latent and transformed Gaussian processes, Water Resources Research, v.48, W01523, doi:10.1029/2011WR011105, 2012.

LI, CHAO; SINGH, VIJAY P.; MISHRA, ASHOK K. Simulation of the entire range of daily precipitation using a hybrid probability distribution. Water Resources Research, v. 48, W03521, doi:10.1029/2011WR011446, 2012.

LUNDGREN, W. J. C.; SOUSA, I. F; NETTO, A. O. A. Uso de distribuições de probabilidades para ajuste aos dados de precipitação mensal do estado de sergipe. Revista Brasileira de Geografia Física, v. 08, n. 01, p. 071-080, 2015.

MELLO, C. R.; SILVA, A. M. Hidrologia: Princípios e aplicações em sistemas agrícolas. Lavras: UFLA, 2013, 455p.

MELLO, Y. R.; OLIVEIRA, T. M. N. Análise estatística e geoestatística da precipitação média para o Município de Joinville (SC). Revista Brasileira de Meteorologia, v. 31, n. 2, p. 229-239, 2016.

MONTGOMERY, D. C.; RUNGER, G. C. Estatística Aplicada e Probabilidade para Engenheiros. 5a Ed., Rio de Janeiro: LTC, 2012, 548p.

MORETTIN, P. A.; BUSSAB, W. O. Estatística Básica. 6a. ed. rev. atual. São Paulo: Saraiva, São Paulo, 2010.

NAGHETTINI, M.; PINTO, E. J. A. Hidrologia estatística. Belo Horizonte: CPRM. 2007, 561p.

NETO, D. D.; ASSIS, J.; TIMM, L. C.; MANFRON, P. A.; SPAROVEK, G.; MARTIN, T. N. Ajuste de Modelos de Distribuição de Probabilidade a Séries Históricas de Precipitação Pluvial Diária em Piracicaba- SP. Revista Brasileira Agrometeorologia, v. 13, n. 2, p. 273-283, 2005.

R CORE TEAM (2017).R: A language and environment for statistical computing. R Foundation for Statistical Computing, Vienna, Austria. ISBN 3-900051-07-0. URL http://www.R-project.org/. 2017.

RONDON, M. A. C. Espacialização de intensidades pluviométricas de chuvas intensas em Mato Grosso do Sul. Dissertação de Mestrado do Programa de Pósgraduação em Tecnologias Ambientais da Universidade Federal de Mato Grosso do Sul, 2001.

SANTOS, E. B.; LUCIO, P. S.; SILVA, C. M. S. Estimating return periods for daily precipitation extreme events over the Brazilian Amazon. Theoretical and Applied Climatology. v. 126, p.585-595, 2016. DOI 10.1007/s00704-015-1605-9 
SOUZA, V. A. S. Eventos de precipitação extremas na Amazônia Ocidental: Rondônia - Brasil. Ji-Paraná: UNIR, Monografia Graduação. 2012, 73p.

SOUZA, V. A. S.; NUNES, M. L. A.; FRANCENER, S. F.; ROSA, A. L. D. Análise da adequação de eventos de precipitação extrema na Amazônia Ocidental em modelos estáticos: Rondônia. Revista Verde de Agroecologia e Desenvolvimento Sustentável. v. 10, n.3, p 13-20, 2015.

SU, B.; KUNDZEWICZ, Z.; JIANG, T. Simulation of extreme precipitation over the Yangtze River Basin using Wake by distribution. Theoretical and Applied Climatology, v. 96, n. 3-4, p. 209-219, 2009.

THEBALDI, M. S. Análise estatística da precipitação máxima diária anual da cidade de Uberaba e vazão mínima diária anual do Rio Uberaba. Revista Agrogeoambiental, v. 4, n. 3, 2012. http://dx.doi.org/10.18406/2316$1817 v 4 n 32012470$

WILKS, D. S. Statistical methods in the atmospheric sciences. $2^{a}$.ed. San Diego: Academic Press, 2006, 629 p. 